\title{
Advances in Modelling Connected and Automated Vehicles
}

\author{
Xiaobo Qu, ${ }^{1}$ Xiaopeng $\mathrm{Li}^{2}{ }^{2}$ Meng Wang, ${ }^{3}$ and Vinayak Dixit ${ }^{4}$ \\ ${ }^{1}$ School of Civil and Environmental Engineering, University of Technology Sydney, Sydney, NSW, Australia \\ ${ }^{2}$ Department of Civil and Environmental Engineering, University of South Florida, Tempa, FL, USA \\ ${ }^{3}$ Department of Transport \& Planning, Delft University of Technology, Delft, Netherlands \\ ${ }^{4}$ School of Civil and Environmental Engineering, University of New South Wales, Sydney, NSW, Australia
}

Correspondence should be addressed to Xiaobo Qu; xiaobo.qu@uts.edu.au

Received 1 November 2017; Accepted 2 November 2017; Published 19 November 2017

Copyright (C) 2017 Xiaobo Qu et al. This is an open access article distributed under the Creative Commons Attribution License, which permits unrestricted use, distribution, and reproduction in any medium, provided the original work is properly cited.

Recent developments of V2V, V2I, and vehicle automation technologies have enabled the concept of the connected and/or automated vehicles (CAVs) to be tested and explored in practice. Connected vehicles are those that communicate with neighbouring vehicles (V2V) and nearby infrastructure (V2I), while automated vehicles are those partially or completely controlled by computers with less or no intervention from human drivers. It is predicted that very soon CAVs will share transport infrastructure with conventional vehicles, which may fundamentally revolutionize our transport systems. With coordination, cooperation, and automation, many believe that CAVs hold promise to improve transport safety, efficiency, and sustainability. However, detailed modelling and technical paths for our existing transport systems to fully capitalize on the CAV concept are yet to be explored, investigated, and examined. It is imperative to develop innovative ideas of utilizing CAVs technologies to improve our transport systems from various aspects, scales, and dimensions. This special issue is focused on the recent advances in modelling connected and automated vehicles. 36 papers were submitted to this special issue, 14 of which were accepted for publication. As the guest editors of this special issue, we would like to summarize the 14 accepted papers below.

With regard to "Modeling Travel Time Reliability of Road Network Considering Connected Vehicle Guidance Characteristics Indexes" by J. Wang et al., travel time reliability has been widely used to evaluate the performance of a transport network. With real-time traffic guidance system, the reliability can be largely improved. In this research, the authors propose a new travel time reliability model using a multilogistic regression approach to account for five characteristics of connected vehicles. The results show that higher penetration rate and compliance rate of connected vehicles will lead to significant improvement of travel time reliability.

"A Trial-and-Error Method with Autonomous Vehicleto-Infrastructure Traffic Counts for Cordon-Based Congestion Pricing" by Z. Liu et al., the authors propose a practical trial-and-error method to solve the optimal toll design problem of cordon-based pricing, where only the traffic counts autonomously collected on the entry links of the pricing cordon are needed. With the fast development and adoption of vehicle-to-infrastructure (V2I) facilities, it is very convenient to autonomously collect these data. A standard stochastic user equilibrium (SUE) with asymmetric link travel time functions is used to assess each feasible toll pattern.

In "The Effect of Connected Vehicle Environment on Global Travel Efficiency and Its Optimal Penetration Rate" by R. Dai et al., the authors examine the effect of connected vehicle environment and the relationship between the penetration rate of connected vehicles and its efficiency. A classical tworoute network is used to conduct a case study with two types of drivers: informed and uninformed drivers. The authors further analyse the merits and demerits of the connected vehicle environment. Different scenarios are subsequently simulated and the results are compared. The findings of this research can be used to better understand the effect of vehicle connectedness and automation in future.

In "Developing a Distributed Consensus-Based Cooperative Adaptive Cruise Control System for Heterogeneous 
Vehicles with Predecessor Following Topology" by Z. Wang et al., the authors propose a novel cooperative adaptive cruise control (CACC) system and design algorithm and protocols for platoon formation, merging maneuvers, and splitting maneuvers. Each vehicle is assumed to only communicate with its following vehicle to reach consensus of the whole platoon, making the vehicle-to-vehicle (V2V) communication more efficient. A simulation study is carried out under different scenarios and a sensitivity analysis is also conducted on the distributed consensus algorithm.

"Analysis of an Automated Vehicle Routing Problem in Logistics considering Path Interruption" by Y. Zhang et al. is focused on the last-mile problem. The authors modify the conventional vehicle routing problem (VRP). An automated vehicle routing problem with time windows (AVRPTW) model considering path interruption is developed and an improved particle swarm optimisation (PSO) algorithm is used to solve the problem. The results show that it is of considerable significance for the promotion of automated vehicles in last-mile problems.

In "Optimal Signal Design for Mixed Equilibrium Networks with Autonomous and Regular Vehicles" by N. Jiang, a signal design problem is proposed for efficiently managing autonomous vehicles (AVs) and regular vehicles (RVs) simultaneously in transport networks. AVs and RVs are assumed to move on separate lanes and share the green time. This problem is formulated as a bilevel programming model. The lower-level describes a mixed equilibrium problem and the upper-level optimises signal timings to allocate green times for both AVs and RVs. Various signal control strategies are evaluated through numerical examples and some insights are obtained.

"DSRC versus 4G-LTE for Connected Vehicle Applications: A Study on Field Experiments of Vehicular Communication Performance" by $\mathrm{Z}$. Xu et al. is focused on testing connected and automated vehicles. Dedicated short-range communication (DSRC) and 4G-LTE are two widely used candidate schemes for connected vehicle (CV) applications. These two standards are compared in this study. A connected vehicle test-bed is established, which integrates the DSRC roadside units, 4G-LTE cellular communication stations, and vehicular on-board terminals. Results show that 4G-LTE is more preferred for the nonsafety applications, such as traffic information transmission, file download, or Internet accessing, which does not necessarily require the high-speed real-time communication, while, for the safety applications, such as collision avoidance or electronic traffic sign, DSRC outperforms the 4G-LTE.

"Accelerated Lane-Changing Trajectory Planning of Automated Vehicles with Vehicle-to-Vehicle Collaboration" by $\mathrm{H}$. Bai et al. aims to generate an accelerated lane-changing trajectory using vehicle-to-vehicle collaboration (V2VC). A polynomial method and cooperative strategies are used to establish a lane-changing model under different degrees of collaboration of vehicles. A simulation model is developed for the accelerated lane-changing process. Results show that safe accelerated lane-changing trajectories can be achieved with the proposed V2VC.

"A $d \phi$-Strategy: Facilitating Dual-Formation Control of a Virtually Connected Team" by B. Sharma describes the design of new centralized acceleration-based controllers for a multitask problem of motion planning and control of a coordinated lead-carrier team fixed in a dual-formation within an obstacle-ridden environment. A $d \phi$-Strategy is developed to ensure virtual connectivity of the carrier robots to the lead robot. For the first time, a dual-formation control problem of a lead-carrier team of mobile manipulators is considered. Computer simulations are used to demonstrate the strategy.

"Will Automated Vehicles Negatively Impact Traffic Flow?” By S. C. Calvert et al. examines whether the low-level vehicle automation will have negative impact on traffic flow. It has been widely accepted that a long gradual transition will occur from manual driving to automated driving. The authors investigate the potential positive and negative effects using an empirically calibrated and validated simulation experiments. They found that low-level automated vehicles will initially have a minor negative effect on traffic flow and road capacities. According to their experiments, any improvement in traffic flow will only be seen at penetration rates above $70 \%$.

In "Characteristic Analysis of Mixed Traffic Flow of Regular and Autonomous Vehicles Using Cellular Automata" by Y. Liu et al., the authors explore the effects of autonomous vehicles and their penetration on heterogeneous traffic flow dynamics. An improved cellular automata model was employed to simulate the problem. According to the simulation results, autonomous vehicles will improve road capacity and free flow speed; the lane-changing frequency between neighbouring lanes evolves with traffic density along a fundamental-diagram-like curve; and the impacts of autonomous vehicles on the collective traffic flow characteristics are mainly related to their smart maneuvers in lane changing and car following.

In "A Genetic Algorithm Approach for Expedited Crossing of Emergency Vehicles in Connected and Autonomous Intersection Traffic" by Q. Lu and K.-D. Kim, an intersection control algorithm is proposed to determine an efficient vehicle-passing sequence that allows the emergency vehicle to cross an intersection as soon as possible while minimising the negative impact on other vehicles. The efficiency of the proposed approach for expedited crossing of emergency vehicles is validated through comparisons with DICA and a reactive traffic light algorithm through extensive simulations. The results show that the proposed genetic algorithm is able to decrease the travel times of emergency vehicles significantly in light and medium traffic volumes without causing any noticeable performance degradation of normal vehicles.

"Commute Equilibrium for Mixed Networks with Autonomous Vehicles and Traditional Vehicles" by Y. Ji et al. is focused on the commute equilibrium for mixed networks with autonomous vehicles and traditional vehicles. A traditional bottleneck model is used to investigate the user equilibrium traffic pattern considering both autonomous vehicles and traditional vehicles. Numerical examples are provided to demonstrate the usefulness of the proposed model. Sensitivity analyses are conducted to show the impact of autonomous vehicles' fees on the traffic pattern and travel costs. 
"PaTAVTT: A Hardware-in-the-loop Scaled Platform for Testing Autonomous Vehicle Trajectory Tracking" by Z. Xu et al. is focused on the testing connected and automated vehicles. Due to the high cost and low flexibility of fullscale test site, a hardware-in-the-loop (HIL) scaled platform was developed in Chang'an University. The system consists of scaled autonomous vehicles, scaled roadway, monitoring centre, transmission device, positioning device, and computers. A high-speed U-turn case is used to test the reliability of trajectory control in this scaled platform. The experiment results demonstrate the effectiveness of the HIL scaled platform.

Taken together, these 14 papers point to three key directions of modelling connected and automated vehicles (i.e., testing, trajectory control and planning, and network optimisation) and applications to special vehicles.

Xiaobo Qu Xiaopeng Li Meng Wang Vinayak Dixit 


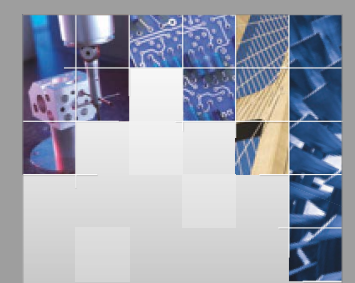

\section{Enfincering}
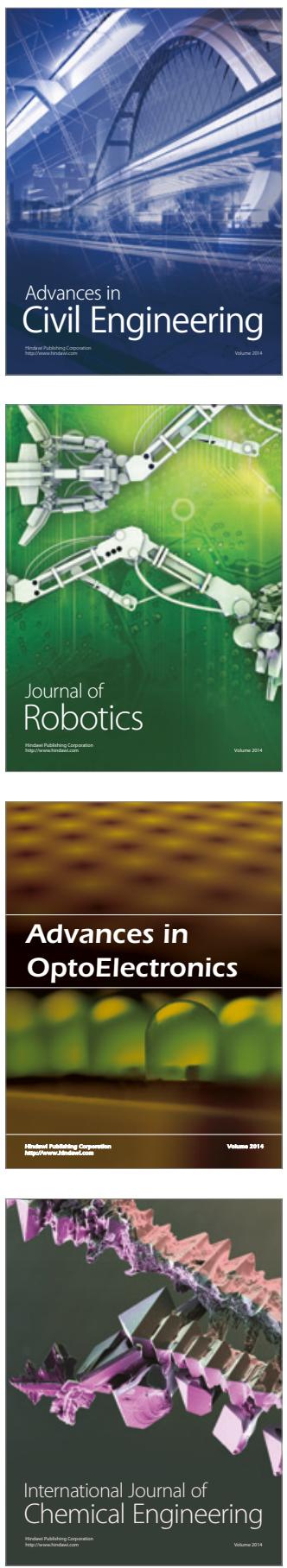

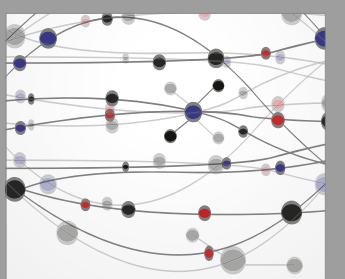

The Scientific World Journal

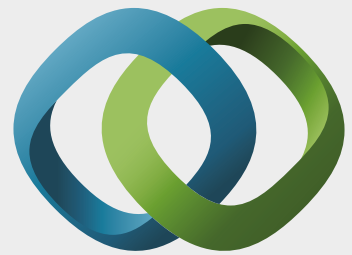

\section{Hindawi}

Submit your manuscripts at

https://www.hindawi.com
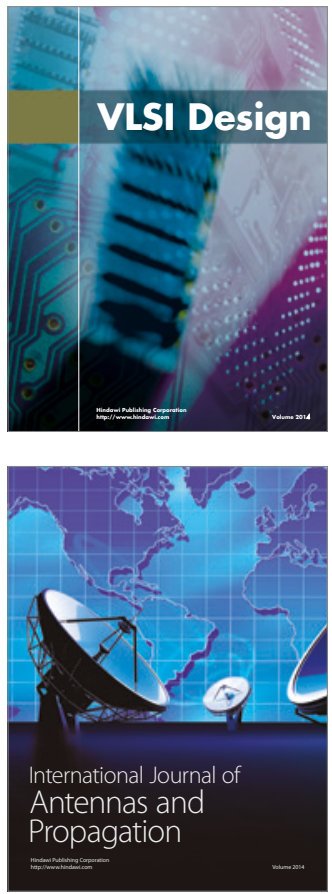

\section{Rotating}

Machinery
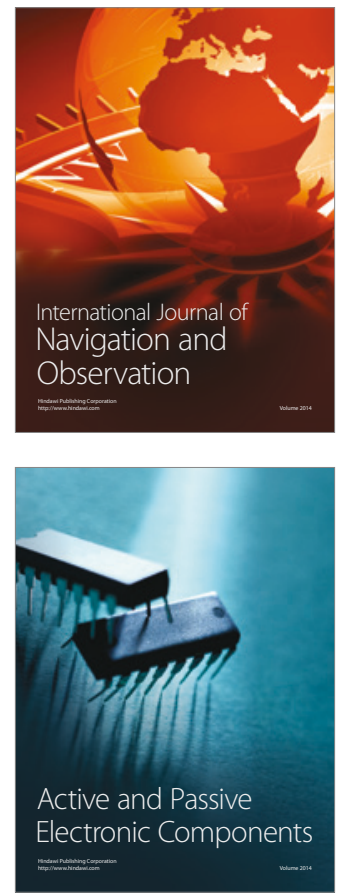
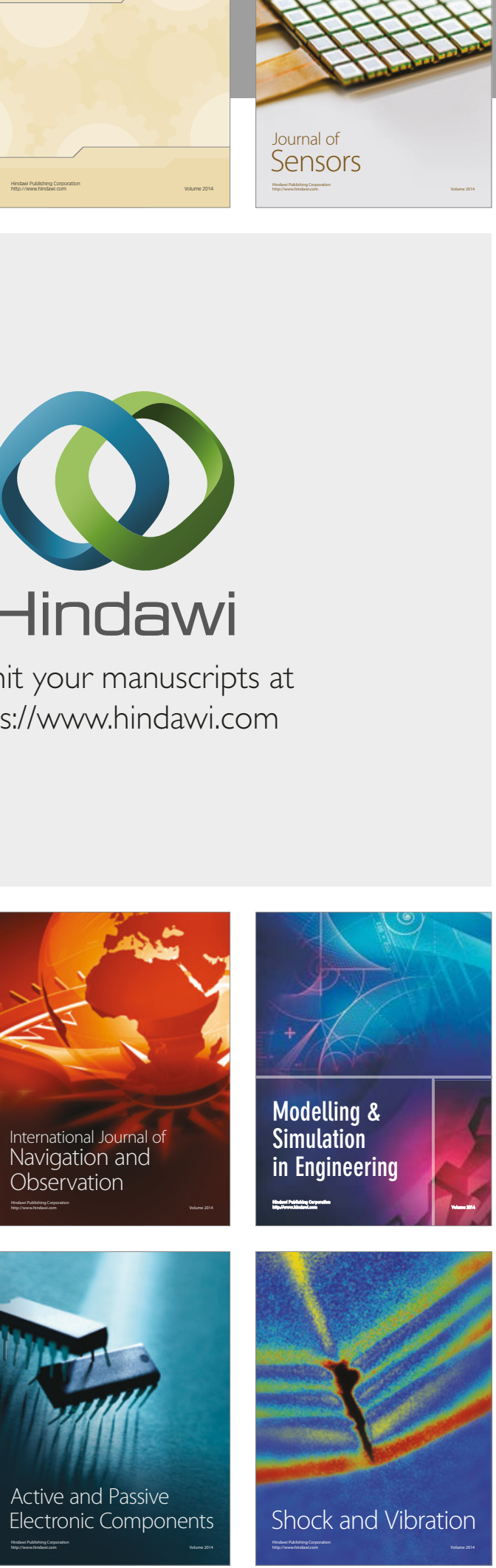
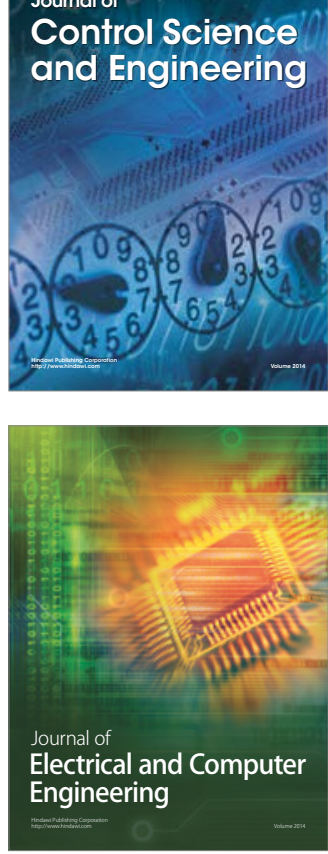

Distributed

Journal of

Control Science

and Engineering
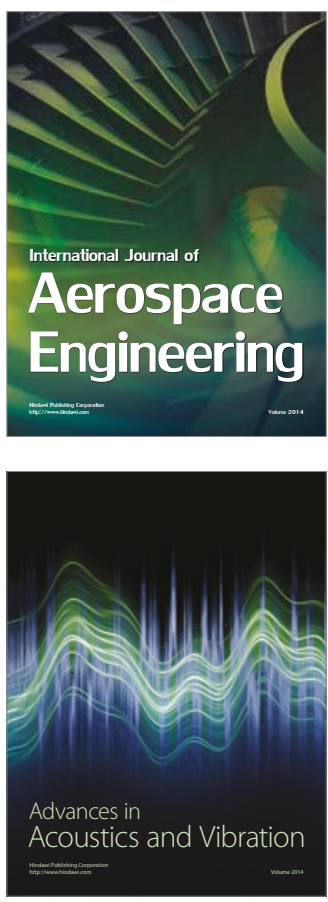

Sensor Networks 